\title{
INTERVENCIÓN COGNITIVO-CONDUCTUAL PARA DISMINUIR DEPRESIÓN Y ANSIEDAD EN UNA PERSONA CON ASMA: ESTUDIO DE CASO
}

\author{
Isaías Vicente Lugo González y Yuma Yoaly Pérez Bautista \\ Facultad de Estudios Superiores Iztacala, UNAM \\ México
}

\begin{abstract}
RESUMEN
Las intervenciones psicológicas en personas con asma van dirigidas a mejorar la adaptación a la enfermedad y disminuir sus consecuencias emocionales. Se presenta un estudio de caso sistemático de una mujer con asma, ansiedad y depresión. El propósito de la intervención fue modificar la percepción de la enfermedad y disminuir los síntomas de ansiedad y depresión. La evaluación se basó en el análisis funcional de la conducta, el cuestionario breve de percepción de enfermedad y la escala de ansiedad y depresión hospitalaria. Los resultados se valoraron con el cambio clínico objetivo y mostraron una modificación clínicamente significativa en la percepción emocional de la enfermedad $(-100 \%)$, consecuencias percibidas $(-85 \%)$ y control personal percibido (100\%). Además, decrementó el nivel de ansiedad (-45\%) y depresión (-75\%). Se concluye que las intervenciones psicológicas deben formar parte del tratamiento multidisciplinario para favorecer una mejoría en calidad de vida y minimizar sus repercusiones emocionales.
\end{abstract}

Palabras Clave:

asma, depresión, ansiedad, percepción de enfermedad, estudio de caso sistemático

\section{COGNITIVE-BEHAVIORAL INTERVENTION FOR REDUCE DEPRESSION AND ANXIETY IN A PERSON WITH ASTHMA: CASE STUDY}

\begin{abstract}
Psychological interventions in people with asthma are aimed at improving adaptation to the illness and reducing its emotional consequences. A systematic case study of a woman with asthma, anxiety and depression is presented. The purpose of the intervention was to modify the illness perception and reduce the symptoms of anxiety and depression. The evaluation was based on the Functional Analysis of Behavior, the Brief Illness Perception Questionnaire and the Hospital Anxiety and Depression Scale. The results were evaluated with the Objective Clinical Change and showed a clinically significant modification in the emotional perception of the illness $(-100 \%)$, perceived consequences $(-85 \%)$ and perceived personal control $(100 \%)$. In addition, the level of anxiety $(-45 \%)$ and depression $(-75 \%)$ decreased. It is concluded that psychological interventions should be part of the multidisciplinary treatment to favor an improvement in quality of life and minimize its emotional repercussions.
\end{abstract}

Keywords:

asthma, depression, anxiety, illness perception, systematic case study

Bitácora del Artículo:

| Recibido: 8 de Septiembre de 2020 | Aceptado: 03 de mayo de 2021 | Publicado en línea: Julio - Diciembre de 2021 | 
Estudio de caso | Intervención Cognitivo-Conductual para disminuir depresión...| Lugo-González \&Z Pérez-Bautista

\title{
Autoría y Derechos de Propiedad Intelectual
}

\section{INTERVENCIÓN COGNITIVO-CONDUCTUAL PARA DISMINUIR DEPRESIÓN Y ANSIEDAD EN UNA PERSONA CON ASMA: ESTUDIO DE CASO}

\author{
Isaías Vicente Lugo González y Yuma Yoaly Pérez Bautista \\ Facultad de Estudios Superiores Iztacala, UNAM \\ México
}

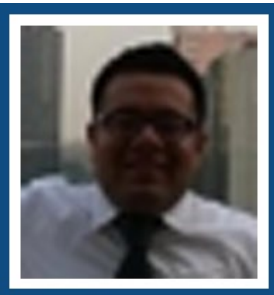

Isaías Vicente Lugo González

FES- Iztacala, UNAM

Correo: isaiasvlg@comunidad.unam.mx

Licenciado y maestro en Psicología por Facultad de Estudios Superiores Iztacala de la UNAM. Estuvo adscrito a la coordinación de psicología de las unidades privadas de hemodiálisis del Centro de Diagnóstico Ángeles, pertenecientes al Grupo Ángeles servicios de salud, y trabajó en el área de Psicología del Centro Médico ISSEMyM Ecatepec.

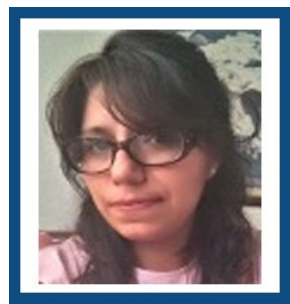

Yuma Yoaly Pérez Bautista

FES- Iztacala, UNAM

Correo:yoalyyu@comunidad.unam.mx

Doctora en Psicología y Salud, por la Facultad de Estudios Superiores Iztacala de la UNAM. Actualmente colabora en la investigación e intervención de problemas de salud en el Grupo de Investigación en Psicología y Salud Sexual de la FES Iztacala. Tiene experiencia docente a nivel licenciatura en la FES Iztacala. Ha publicado en revistas indexadas a nivel nacional e internacional; es coautora de capítulos de libro.

\section{CONTRIBUCIÓN DE LOS AUTORES}

Isaías Vicente Luego González estuvo a cargo de la aplicación del tratamiento, análisis de datos, redacción del manuscrito | Yuma Yoaly Pérez Bautista participó en la discusión de la intervención, análisis de datos, redacción del manuscrito.

\section{AGRADECIMIENTOS}

Se agradece I Instituto Nacional de Enfermedades Respiratorias por las facilidades para llevar a cabo la intervención.

\section{DATOS DE FiLIACIÓN dE los Autores}

Unidad de Investigación Interdisciplinaria en Ciencias de la Salud y Educación (UIICSE). Facultad de Estudios Superiores Iztacala. UNAM

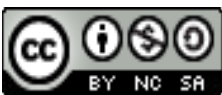

Copyright: (C) 2021 Lugo-González, I. y Pérez-Bautista, Y. Y.

Este es un artículo de acceso abierto distribuido bajo los términos de la licencia Creative Commons Reconocimiento-NoComercial 4.0 Internacional, por lo que su contenido gráfico y escrito se puede compartir, copiar y redistribuir total o parcialmente sin necesidad de permiso expreso de sus autoras con la única condición de que no se puede usar con fines directamente comerciales y los términos legales de cualquier trabajo derivado deben ser los mismos que se expresan en la presente declaración. La única condición es que se cite la fuente con referencia a la Revista Digital Internacional de Psicología y Ciencia Social y a sus autoras. 


\section{TABLA DE CONTENIDO}

Identificación del caso, 368

Análisis del motivo de la intervención, 368

Antecedentes del problema, 368

Descripción y análisis del problema, 368

\section{PLAN DE INTERVENCIÓN}

Descripción del plan intervención, 369

Establecimiento de los objetivos del plan de intervención, 369

Técnicas de evaluación del plan de intervención, 370

\section{EFICACIA DEL PLAN DE INTERVENCIÓN}

Aplicación del plan de intervención, 370

\section{PRESENTACIÓN DE RESULTAdos}

Análisis de los cambios que produjo el plan de intervención, 372

Significancia clínica de los cambios, 373

Relevancia social del cambio, 373

Pronóstico

Descripción de la fase de seguimiento, 374

Resultados en la fase de seguimiento, 374

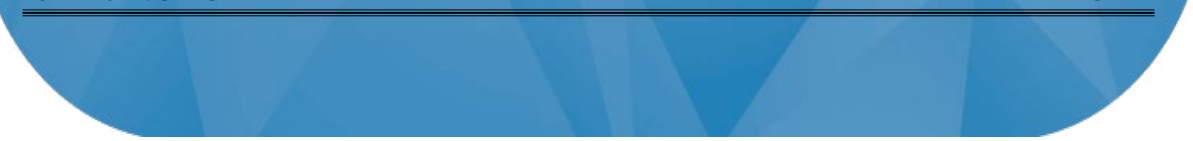




\section{INTRODUCCIÓN}

$\mathbf{L}$ as enfermedades crónico degenerativas se caracterizan por ser de larga duración, así como por carecer de cura, lo que condiciona usar tratamientos para el control de síntomas y un ajuste constante a la enfermedad (Barley y Lawson, 2016; Bravo-González, 2014; Grau-Abalo, 2016; Larsen, 2009).

Vivir con una enfermedad crónico degenerativa se ha asociado con el desarrollo de síntomas de ansiedad y depresión (Katon, 2011; Gerontoukou, Michaelidoy, Rekleiti, Saridi, y Souliotis, 2015). En el caso de las enfermedades respiratorias como el asma, estas sintomatologías están asociadas con la intensidad y frecuencia de los síntomas de la enfermedad (disnea, opresión torácica, etcétera) y a las consecuencias de un control inadecuado de ésta (limitaciones funcionales, mayor frecuencia de síntomas diurnos, nocturnos, necesidad de medicación de rescate y atención de urgencias); Foster, Lavoie, y Bouler, 2011; Global Initiative for Asthma [GINA], 2019).

Se conoce que entre 1 y $48 \%$ de los pacientes con asma presentan algún grado de ansiedad y depresión (Ciprandi, Schiavetti, Rindone y Ricciardolo, 2015; Di Marco et al., 2010; Ritz, Meuret, Trueba, Fritzsche y Von Leupoldt, 2013). Debido a lo anterior, dicha sintomatología ha sido ampliamente investigada en este tipo de pacientes porque son las comorbilidades psicológicas más prevalentes y guarda relación con la calidad de vida, limitaciones funcionales y el control de la enfermedad (Di Marco et al., 2010; Foster et al., 2011; Yorke, Fleming, Shuldham, Rao, y Smith, 2015).

Como ejemplo de lo anterior, Ciprandi et al. (2015) desarrollaron una investigación con 263 pacientes con asma, identificando que cerca de $37 \%$ presentaba ansiedad y $11 \%$ depresión. Por su parte, Coban y Aydemir (2014) evaluaron a 174 pacientes, donde se observó que $33 \%$ presentó ansiedad y casi $48 \%$ depresión. Ryu, Chun, Lee y Chang (2010) evaluaron a 227 pacientes con tres tipos de enfermedades respiratorias, encontrando que de los pacientes con asma $(n=37) 16 \%$ presentó síntomas de ansiedad y $41 \%$ sintomatología moderada y severa de depresión. Por último, Di Marco et al. (2010) reportaron que, en una muestra de 315 pacientes hospitalizados, 39\% calificó con datos para ansiedad y $27 \%$ para depresión.

Ahora bien, es importante considerar que, en el caso de México, los síntomas de ansiedad presentes en la población con asma se han reportado entre 50 y 75\%, mientras que los de depresión oscilan entre 30 y 50\% (Espinosa et al., 2016; Guillen, González, Sánchez y Fernández-Vega, 2018; Guillen y González, 2019; Lugo-González, Reynoso-Erazo y Fernández-Vega, 2014).

Es evidente el impacto emocional que genera la enfermedad y el efecto negativo de la depresión y ansiedad en el manejo de ésta (Ciprandi et al., 2015; Coban y Aydemir, 2014; Di Marco et al., 2010), por lo que un abordaje adecuado para los pacientes corresponde con tratamientos multidisciplinarios que incluyen intervenciones psicológicas y tratamiento médico (DeGood, Crawford y Jongsma, 1999; GINA, 2019).

En las intervenciones psicológicas desde la medicina conductual en asma, los objetivos particulares corresponden con 1) favorecer la prevención de crisis de asma; 2) mejorar la función pulmonar; 3) aumentar la calidad de vida; 4) incrementar el control del asma, y 5) disminuir el impacto emocional de la enfermedad (DeGood, Crawford y Jongsma, 1999; Guillen y Lugo-González, 2014; Ritz et al., 2013).

Estas intervenciones pueden estar conformadas por diversos componentes de tratamiento como: estrategias psicoeducativas, técnicas para favorecer la relajación y/o incrementar el afrontamiento funcional frente al impacto físico y emocional de la enfermedad (DeGood et al., 1999; Guillen y Lugo-González, 2014; Ritz et al., 2013; Yorke et al., 2015).

Desde esta lógica, los mecanismos de intervención pueden estar orientados hacia los componentes cognitivos, porque una evaluación o percepción negativa de la enfermedad favorece repercusiones emocionales y limitaciones en el manejo de la enfermedad (Achstetter, Schultz, Faller y Schuler, 2016; Bahçecioğlu y Çevikakyl, 2014; Lugo-González et al., 2014), por lo que el uso de técnicas cognitivas para favorecer una modificación en la percepción de la enfermedad son un recurso ideal. Sin embargo, también podría ser útil el uso de técnicas conductuales con las que, mediante procedimientos como los experimentos o ensayos conductuales, se exponga a las personas a tener contacto con consecuencias positivas, contrarias a lo esperado y que resulten en cambios cognitivos o perceptuales (Beck, Rush, Shaw y Emery 2010; McAndrew et al., 2008).

En este sentido se podría intervenir, de manera inicial, con estrategias conductuales orientadas a favorecer el afrontamiento activo de los problemas vinculados con la enfermedad, ayudando a la modificación de percepciones negativas de la enfermedad, cambios en las repercusiones emocionales y adaptación al padecimiento (Freitas et al., 2019; Karekla, Karademas y Gloster, 2019; Lugo-González y Pérez-Bautista, 2018; Prapa et al., 2017). 
Ahora bien, la evidencia más actual respecto de la efectividad de las intervenciones psicológicas cognitivo conductuales y de medicina conductual en pacientes con asma ha resultado ser de leve a moderada en cuando al manejo de ansiedad y depresión (Pateraki y Graham, 2018; Smith y Jones, 2015). A pesar de esto, se han propuesto como formas de intervención complementarias a los tratamientos médicos para el manejo del impacto emocional, afrontamiento y adaptación a la enfermedad, calidad de vida, adherencia al tratamiento y control del asma, interviniendo sobre todo con técnicas basadas en elementos educativos, estrategias para favorecer la relajación, solución de problemas, afrontamiento activo y las enfocadas a la modificación de la percepción de la enfermedad (Kew, Nashed, Dulay y Yorke, 2016; Pateraki et al., 2018; Ritz et al., 2013; Smith y Jones, 2015; Stanescu, Kirby, Thomas, Yardley y Ainsworth, 2019; Yorke et al., 2015), concentrándose en abordajes individuales y en los mecanismos de cambio y mantenimiento de los problemas emocionales (Pateraki et al., 2018; Ritz et al., 2013; Smith y Jones, 2015).

\section{MÉTodo}

\section{Identificación del caso}

Con base en la estructura metodológica de Iwakabe y Gazzola (2009), se muestra un estudio de caso sistemático en el que participó Sandra (nombre ficticio), mujer adulta de 30 años, con sintomatología de ansiedad, depresión y con un diagnóstico médico de asma de difícil control. Sandra es casada desde hace dos años, no tiene hijos y vive en la casa de sus padres, junto con su esposo. Tiene escolaridad técnica (optometría) y es originaria y residente del Estado de México.

La intervención se efectuó en el Instituto Nacional de Enfermedades Respiratorias (INER) y se derivó de un protocolo de investigación de percepción de enfermedad, adherencia al tratamiento y control del asma, evaluado y aceptado por el comité de ética en investigación del INER (núm. C47-18).

\section{Análisis del motivo de la intervención}

Sandra acude a consulta psicológica por decisión propia (posterior a responder una evaluación), debido a que tenía cuatro meses experimentando malestar emocional (sintomatología de ansiedad y depresión) asociado a las repercusiones de vivir con asma, las consecuencias en su vida cotidiana, la experimentación de síntomas, las limitaciones funcionales, las visitas al hospital, la posibilidad de ser hospitalizada o permanecer en urgencias, y la realización de estudios (espirometría y su posterior experiencia de síntomas).

\section{Antecedentes del problema}

Sandra fue diagnosticada con asma desde los tres años (27 años con la enfermedad); acudió a un hospital especializado en enfermedades respiratorias en la Ciudad de México desde los nueve y hasta los 16 años, periodo en que no efectuó un tratamiento de control para el asma, por lo que acudía con frecuencia por crisis de asma, y al hacerse estudios de espirometría, éstas evidenciaban obstrucción bronquial.

Entre los 16 y los 29 años tuvo tres crisis de asma (dos atendidas en hospitales públicos de la Ciudad de México, y en las cuales perdió el conocimiento) que la llevaron a ser hospitalizada; fue en 2018 la más severa, porque estuvo internada durante 11 días; dicha hospitalización se extendió debido a que sus espirometrías evidenciaban obstrucción.

Además de visitar el hospital en tres ocasiones por crisis de asma, la paciente normalmente presentaba síntomas frecuentes, estaba limitada en sus actividades cotidianas, tenía problemas para dormir y usaba de manera excesiva el medicamento de rescate (salbutamol), condiciones que repercutían de modo cotidiano en diferentes ámbitos de su vida.

\section{Descripción y análisis del problema}

Con base en la información recabada se diseñó el análisis funcional de la conducta (AFC), utilizando un modelo ABC (Antecedente-Conducta-Consecuencia; Kanfer y Saslow, 1965), el cual mostró que Sandra acudió por malestar emocional asociado al impacto de vivir con asma, mismo que se manifestó en la presencia de ansiedad y depresión desde hacía cuatro meses, al tener una crisis de asma, y estar hospitalizada durante 11 días porque sus espirometrías evidenciaban obstrucción, razón por la cual no podía ser dada de alta. Durante dichos meses tuvo que acudir a consultas y a hacerse más estudios, mismos que tenían un resultado adverso y lo cual la colocaba en posibilidad de ser evaluada en urgencias y/o ser hospitalizada.

Aunado a lo anterior, presentaba respuestas anticipatorias de ansiedad asociadas con una percepción negativa de la enfermedad. Otros sucesos relacionados con estas problemáticas son sus experiencias con el diagnóstico temprano de asma, pérdida de conocimiento, hospitalizaciones de larga duración, limitaciones funcionales, uso excesivo de salbutamol y evidencia frecuente de obstrucción en estudios, todo ello por un bajo control de la enfermedad.

El mantenimiento de estas problemáticas es consecuencia de la dificultad para lograr — a pesar de encon- 
Estudio de caso | Intervención Cognitivo-Conductual para disminuir depresión... | Lugo-González \& Q Pérez-Bautista

trarse en tratamiento- un control óptimo de la enfermedad, uso de medicamento de rescate, presencia de síntomas de asma, limitaciones funcionales, evitación de situaciones sociales y físicas, y una continua valoración negativa de la enfermedad, además de su perspectiva para abordar sus problemas (orientación negativa), así como una tendencia a afrontarlos de manera evitativa. El AFC se muestra gráficamente en la figura 1.

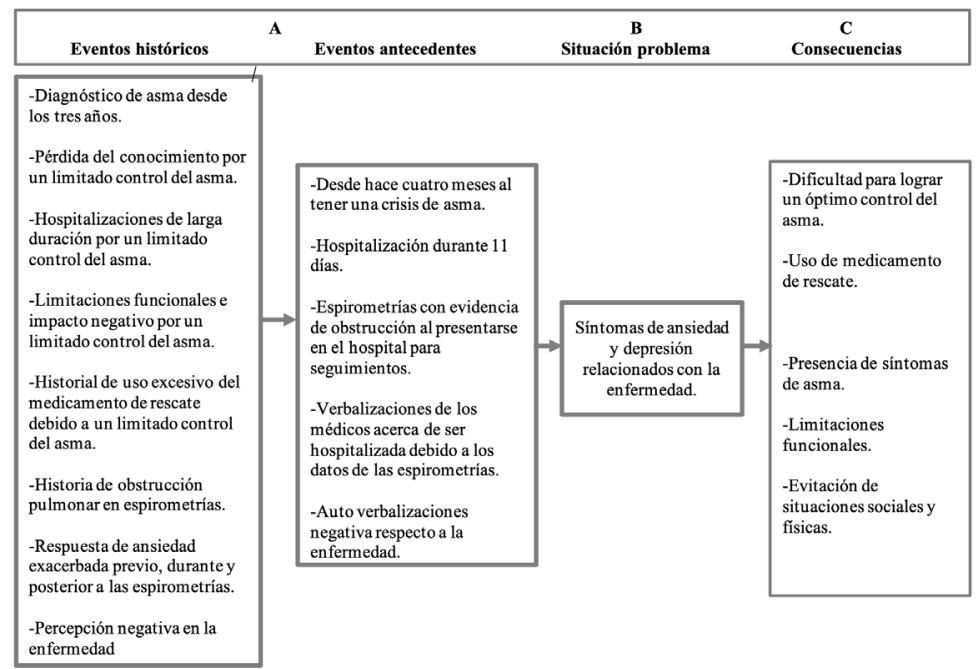

Figura 1.

AFC de Sandra.

\section{Plan de intervención}

\section{Descripción del plan intervención}

Derivado del AFC y la evaluación psicométrica se diseñó una intervención cognitivo-conductual enfocada en las estrategias de psicoeducación, solución de problemas, planeación de actividades y revaloración cognitiva. El tratamiento estuvo estructurado de la siguiente manera: evaluación inicial, intervención, evaluación final y seguimiento. En total se efectuaron tres sesiones de evaluación y seis sesiones de intervención, con una duración de entre 60 y 90 minutos cada una. Las variables de la intervención se describen en la tabla 1.
Tabla 1.

Descripción de técnicas de intervención para el caso de Sandra.
TÉCNICA

DescripCión

Estrategia enfocada en la transmisión de información respecto a una conducta, enfermedad y/o tratamiento,

Psico educación con el objetivo de lograr un cambio comportamental y/o de creencias asociadas a ésta. Puede ser dirigida por un especialista y efectuada por diferentes medios (verbal, práctica conductual, con material impreso o audiovisual) (Colom, 2011).

Estrategia utilizada para favorecer las habilidades de plantear, analizar y poner en marcha planes de acción para resolver Solución de problemas problemas de manera organizada, estructurada y mediante una serie de pasos que permita un cambio conductual dirigido a objetivos específicos (Nezu, Nezu y D'Zurilla (2007)

\section{.Estrategia enfocada en la} sistematización de actividades Planeación potencialmente reforzantes dirigidas de a favorecer la implementación de actividades estrategias para afrontar situaciones de riesgo para el estado de ánimo (Kanter et al., 2010).

Estrategia COMPLeMENTARIA (VER DesarRollo del CASO)

Estrategia orientada a modificar formas de pensamiento y percepción asociadas de manera negativa con una situación,

Revaloración Cognitiva enfermedad o tratamiento, considerando que estas se asocian con experiencias emocionales y formas de comportamiento específicas (Beck et al., 2010).

\section{Establecimiento de los objetivos del plan de intervención}

El propósito principal de la intervención cognitivo-conductual fue modificar la percepción de la enfermedad y disminuir la sintomatología de ansiedad y depresión por medio de la aplicación de técnicas para favorecer el afrontamiento funcional y la exposición a condiciones diferentes a las percibidas, esto debido a las relaciones identificadas en el AFC. La definición conceptual y operacional se muestran en la tabla 2 . 
Tabla 2.

Definición conceptual y operacional de las variables de resultados para el caso de Sandra.

$\begin{array}{ccc}\text { Variables } & \text { DefiniCión } & \text { Definición } \\ \text { CONCEPTUAL } & \text { OPERACIONAL }\end{array}$

\begin{tabular}{|c|c|c|}
\hline $\begin{array}{l}\text { Percepción de } \\
\text { enfermedad }\end{array}$ & $\begin{array}{l}\text { Percepción de } \\
\text { temporalidad, } \\
\text { identidad, control, } \\
\text { consecuencias, } \\
\text { coherencia } \\
\text { y resultados } \\
\text { emocionales } \\
\text { atribuidos a la } \\
\text { enfermedad } \\
\text { (Leventhal, Leventhal } \\
\text { y Contrada, 1998). }\end{array}$ & $\begin{array}{l}\text { Cuantificación } \\
\text { obtenida en los ítems } \\
\text { del B-IPQ (Broadbent, } \\
\text { Petrie, Main, y } \\
\text { Weinman, 2006). }\end{array}$ \\
\hline $\begin{array}{l}\text { Síntomas de } \\
\text { ansiedad }\end{array}$ & $\begin{array}{l}\text { Respuestas } \\
\text { cognitivas, } \\
\text { conductuales } \\
\text { y emocionales, } \\
\text { caracterizadas por } \\
\text { anticipación al peligro } \\
\text { y activación elevada } \\
\text { (Barragán, Parra, } \\
\text { Contreras y Pulido, } \\
\text { 2003). }\end{array}$ & $\begin{array}{l}\text { Cuantificación } \\
\text { obtenida en la escala } \\
\text { de ansiedad del HADS } \\
\text { (Zigmond y Snaith, } \\
\text { 1983). }\end{array}$ \\
\hline
\end{tabular}

\section{Respuestas}

cognitivas,

conductuales y emocionales que incluyen sentimientos

Síntomas de de tristeza, depresión pensamientos

con tendencia

hacia lo negativo

y deterioro en el

plano motivacional

(Vázquez, 2002).
0-10, lo que se traduce en que a mayor puntuación en cada ítem mayor es la característica medida; por ejemplo, mayores consecuencias percibidas, control personal o impacto emocional. La escala tiene datos adecuados de confiabilidad test-retest oscilando entre $r=0.42$ y $r=0.72$ con un intervalo de seis semanas entre aplicaciones; además tiene evidencias de validez de tipo predictivo, convergente y divergente.

- Escala de Ansiedad y Depresión Hospitalaria (HADS) (Zigmond y Snaith, 1983): Constituida por 14 ítems; siete para síntomas de ansiedad y siete para síntomas de depresión. Tiene la finalidad de evaluar dicha sintomatología en personas encontradas en el contexto hospitalario. Se recomienda su uso hospitalario porque excluye sintomatología somática de ansiedad y depresión. Se responde en una escala tipo Likert de 0 a 3 puntos dependiendo de la intensidad de los síntomas. Las puntuaciones de la escala van de 0 a 21 puntos para ambos tipos de síntomas y se han configurado como puntos de corte los siguientes: 0-7 síntomas no significativos; 8-10 síntomas dudosos, y 11-21 síntomas presentes. En México cuenta con adecuadas propiedades psicométricas de confiabilidad en pacientes con obesidad (Morales, Alfaro, Sánchez, Guevara y Vázquez, 2007), infección por VIH (Nogueda, Pérez, Barrientos, Robles y Sierra, 2013), cáncer (Galindo et al., 2015) y enfermedad renal crónica (Lugo-González, Pérez-Bautista y Sánchez-Cervantes, 2018), con datos de alfa de Cronbach de $0.82,0.90,0.86$ y 0.82 , respectivamente). Manteniendo la estructura de dos factores, con excepción del estudio de Nogueda et al. (2013).

\section{Técnicas de evaluación del plan de intervención}

- Entrevista conductual: Para la exploración de datos generales, motivo de consulta, aspectos asociados a la enfermedad y análisis conductual para el desarroIlo del AFC.

- Brief IIIness Perception Questionnaire (B-IPQ; Broadbent et al., 2006): Instrumento para la evaluación de la percepción de la enfermedad, el cual está constituido por nueve ítems divididos de la siguiente manera: 1) consecuencias; 2) temporalidad; 3) control personal de la enfermedad; 4) control del tratamiento; 5) identidad; 6) preocupación por la enfermedad; 7) coherencia (comprensión de la enfermedad); 8) representación emocional de la enfermedad, y 9) causas de la enfermedad. Cada ítem, excepto el 9 ), se responde con una escala continua que va de

\section{Eficacia del plan de intervención}

\section{Aplicación del plan de intervención}

Dada la estructura de evaluación en intervención, para efectuarla se utilizaron los siguientes materiales.

- Manual de solución de problemas para pacientes con asma. Herramienta teórico-práctica acerca del proceso de solución de problemas en el que se abarca la orientación al problema y los cinco pasos para resolver un problema del modelo de Nezu, Nezu y D'Zurilla (2007).

- Formato Haciendo mi plan de actividades. Formato comúnmente utilizado en intervenciones conductuales para depresión basadas en activación conductual. Incluye los siguientes elementos para la planeación de actividades: ¿Qué quiero hacer? ¿Qué 
necesito? ¿Cuándo y cuánto tiempo durará? ¿Lo haré solo o acompañado? ¿Cómo me sentí al hacerlo de 0 a 100? El presente formato se basó en el estudio de Lugo-González y Pérez-Bautista (2016).

Las actividades de evaluación e intervención se describen en la tabla 3.

Tabla 3.

Actividades de evaluación e intervención para el caso de Sandra.

\section{EtAPA/SEsión}

\section{DesCRIPCIÓN DE ACTIVIDADES}

Evaluación

inicial.

Sesión 1

1) entrevista conductual para el desarrollo del AFC, 2) aplicación el HADS y 3) aplicación del $\mathrm{BIPQ}$.

1) retroalimentación del AFC a la paciente y propuesta del plan de intervención (psicoeducación, solución de problemas, planeación de actividades). En este, la paciente encontró congruencia con su perspectiva del problema, el AFC y la propuesta de intervención; 2) inicio de la intervención en solución de problemas, de los componentes de orientación al problema, solución de problemas y estilo de solución de problemas.

1) lista de problemas, definición del problema e identificación de barreras para la solución de $\begin{array}{ll}\text { Intervención. } & \text { problemas; y 2) como actividad para casa se } \\ \text { dejó el planteamiento de posibles soluciones al }\end{array}$ Sesión 3 problema y una lista de actividades potenciales para realizar, que guardaran relaciones con el impacto de su enfermedad.

1) vínculo entre problemas, se valoraron las opciones de solución, 2) se introdujo la planeación de actividades y se planearon actividades para los siguientes 15 días, y 3) como actividad para casa se planteó la realización de actividades planeadas en sesión.

1) aplicación del HADS, 2) aplicación del BIPQ, 3) análisis de logros con planeación de actividades, impacto en las evaluaciones Sesión 5 y mecanismos de impacto, 4) realización del segundo plan de actividades, y 5) como actividad para casa se planteó la realización de actividades planteadas en sesión.

Derivado de una serie de hallazgos relacionados con su enfermedad, su neumóloga decidió proponerle nuevas opciones de diagnóstico y de tratamiento invasivo (termoplastia bronquial) para mejorar su control del asma, mismas que implicaban un nuevo ajuste por parte de Sandra. Por ello, al presentarse un nuevo desajuste emocional se incorporaron estrategias de intervención (no correspondientes al plan original) y se decidió enfocarse en el trabajo propuesto por su médico.
EtAPA/SEsión

Descripción de ACTIVIDADES

1) análisis de logros con planeación de actividades, impacto en las evaluaciones y mecanismos de impacto, 2) realización del

Sesión 6 tercer plan de actividades, y 4) como actividad para casa se planteó la realización de actividades planeadas en sesión. Al finalizar esta sesión se presentó una situación problema. *

1) aplicación del HADS, 2) aplicación del BIPQ, 3) análisis de una nueva situación problema, 4) introducción al modelo $A B C$ para revaloración cognitiva, 4) introducción y

Evaluación/ Intervención. Sesión 7 práctica de estrategia de relajación progresiva de Jacobson, y 5) planeación de actividades sobre búsqueda de información para solucionar y afrontar el nuevo problema.

Sesión 8

1) ejercicios de revaloración cognitiva, 2) práctica de relajación progresiva de Jacobson y 3) análisis de información encontrada.

1) aplicación del HADS, 2) aplicación del BIPQ,

Seguimiento. 3) planteamiento de seguimiento posterior a Sesión 9 la resolución de problemas vinculados con su nuevo abordaje médico.

Derivado de una serie de hallazgos relacionados con su enfermedad, su neumóloga decidió proponerle nuevas opciones de diagnóstico y de tratamiento invasivo (termoplastia bronquial) para mejorar su control del asma, mismas que implicaban un nuevo ajuste por parte de Sandra. Por ello, al presentarse un nuevo desajuste emocional se incorporaron estrategias de intervención (no correspondientes al plan original) y se decidió enfocarse en el trabajo propuesto por su médico.

\section{Presentación de resultados}

Para analizar el efecto de la intervención se usó el índice de cambio clínico objetivo expresado en porcentaje (CCO) (Cardiel, 1994) para la comparación de las medidas de la HADS y el BIPQ respecto de la evaluación inicial y la segunda evaluación (sesión 5), medidas que corresponden con la implementación de la intervención planteada en un inicio (psicoeducación, solución de problemas y planeación de actividades). Es necesario mencionar que este contraste ya no se efectuó para las siguientes mediciones debido a las características del caso (incorporación de situaciones adversas y otras técnicas de intervención).

Se recomienda el CCO para evaluar la significancia clínica de los efectos de un tratamiento, y se reporta que una diferencia entre la posevaluación y la preevaluación dividida entre esta última, se indica que un índice $\geq a$ $20 \%$ es clínicamente significativo; puede haber cambio negativo o positivo, debido al tipo de variable que se esté analizando (García-Flores y Sánchez-Sosa, 2013). 


\section{Análisis de los cambios que produjo el plan de intervención}

Se corroboró que la paciente tenía una orientación negativa hacía los problemas y un estilo de afrontamiento evitativo, por lo que se procedió a trabajar con la identificación de problemas y barreras para el afrontamiento activo y así delinear las soluciones para los problemas.

Al momento de la implementación de la solución de los problemas, la planeación de actividades fungió como vínculo para hacerlo y se consideró una lógica de experimentos conductuales enfocados a conseguir evidencia en contra de la percepción negativa de la enfermedad, y como resultado disminuir el impacto emocional, favorecer el control de ésta y la calidad de vida. En este sentido los resultados iniciales se orientan a la planeación de actividades constituidas como herramientas de cambio cognitivo e implementadas entre las sesiones cuatro y siete (tabla 4).

Tabla 4.

Descripción, planeación y efectos de las actividades a efectuar durante la intervención del caso de Sandra.

\begin{tabular}{|c|c|c|c|c|}
\hline $\begin{array}{c}\text { ¿QUÉ ACTIVIDAD QUIERO } \\
\text { HACER? }\end{array}$ & ¿QUÉ NECESITO? & $\begin{array}{l}\text { ¿CUÁNDO Y CUÁNTO } \\
\text { TIEMPO DURARÁ? }\end{array}$ & $\begin{array}{l}\text { ¿LO HARÉ SOLA O } \\
\text { ACOMPAÑADA? }\end{array}$ & $\begin{array}{l}\text { ¿Cómo me Sentí AL } \\
\text { HACERLo? * }\end{array}$ \\
\hline \multicolumn{5}{|c|}{ Primer PLAN de ACtividades } \\
\hline Hacer ejercicio. & Ropa deportiva. Energía. & $\begin{array}{l}\text { Lunes-viernes, } 30-40 \\
\text { minutos. }\end{array}$ & Sola. & 70 \\
\hline Andar en bicicleta. & Bicicleta. & Diario, 15 minutos. & Sola. & 80 \\
\hline Ir a un concierto. & Boleto. & 1 día, dos horas. & $\begin{array}{l}\text { Acompañada por } \\
\text { esposo y amigos. }\end{array}$ & 90 \\
\hline Bailar. & Pareja, música. & 10 minutos. & Acompañada. & 60 \\
\hline Ir a la feria. & Dinero. & 1 día, tres horas. & Acompañada. & 90 \\
\hline Patinar. & $\begin{array}{l}\text { Patines, coderas, } \\
\text { rodilleras. }\end{array}$ & 1 hora, 1 día. & Acompañada. & 80 \\
\hline
\end{tabular}

SEgundo PLAN DE ACTIVIDAdes

Hacer ejercicio. Ropa deportiva. Energía. Lunes-viernes, 30-40 minutos.

Andar en bicicleta.

Bicicleta.

Diario, 15 minutos.

Sola.

80

Ir a un concierto.

Boleto.

1 día, dos horas.

1 día.

Acompañada por esposo y amigos.

Acompañada.

Acompañada.

Salir con amigos.

Dinero, tiempo.

Medio día.

Tiempo y ánimo.

TERCER PLAN DE ACTIVIDADES

Hacer ejercicio. Ropa deportiva. Energía. Lunes-viernes, 30-40 minutos.

Sola.

60

Andar en bicicleta.

Bicicleta.

Diario, 15 minutos.

Sola.

60

Reunión familiar.

Tiempo y ánimo.

4 horas.

Acompañada.

40

* La valoración correspondió con una escala de 0 a 100, donde un valor más cercano a 100 implica un mejor nivel de estado de ánimo. 
Es necesario indicar que en la planeación dos no pudo efectuar una actividad (salir con amigos) por falta de organización. Asimismo, en el tercer plan las principales actividades que realizó disminuyeron sus propiedades reforzantes (problema relacionado con la enfermedad).

\section{Significancia clínica de los cambios}

Derivado de las experiencias que Sandra tuvo en el proceso de solución de problemas y la planeación de actividades, el resultado fue opuesto a lo que ella esperaba. En este sentido, los cambios (figura 2) en la percepción de enfermedad se evidenciaron en la disminución del impacto emocional asociada a esta $(-100 \%)$, consecuencias negativas percibidas $(-75 \%)$ y aumento en el control percibido de la enfermedad (100\%); siendo clínicamente significativos, dichos cambios no se presentaron en la temporalidad, de tal forma que Sandra siempre consideró al padecimiento como algo crónico.

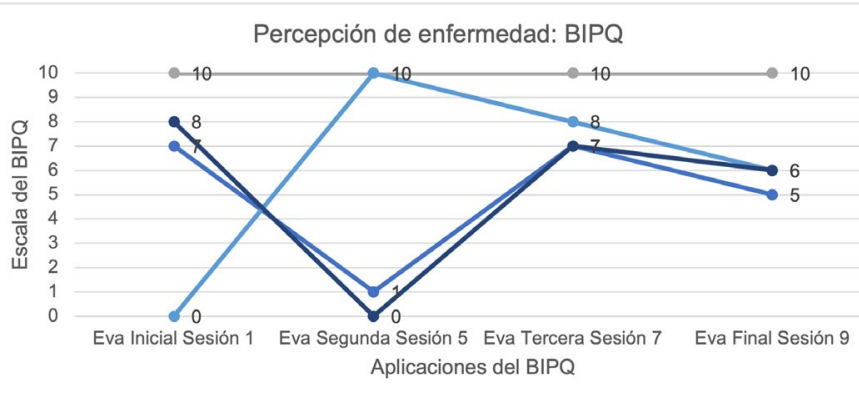

Figura 2.

Resultados en percepción de enfermedad: BIPQ.

A pesar de que después de la quinta sesión aún se continuó con la intervención de plan de actividades, la siguiente evaluación se hizo en dos sesiones después, momento donde se identificaron cambios en el avance de los resultados obtenidos debido a problemas con nuevos datos en el diagnóstico de la enfermedad y formas de tratamiento.

Dadas las características del problema presentado, se decidió incluir a la intervención técnicas complementarias y se consiguió un decremento significativo clínicamente para la evaluación final en las consecuencias percibidas de la enfermedad $(-28 \%)$, no así para el impacto emocional de la enfermedad $(-14 \%)$; al final la percepción de control de la enfermedad continuó disminuyendo $(-40 \%$ al contrastar las sesiones cinco y nueve, $y-25 \%$ entre las sesiones siete y nueve).

Los cambios también se presentaron en las variables de tipo emocional; en la figura 3 se muestra que entre la primera (evaluación inicial) y quinta sesiones (segunda evaluación), la ansiedad disminuyó 45\% y la depresión 75\%, siendo cambios clínicamente significativos. Después de la sesión seis la ansiedad se incrementó en $57 \%$ y la depresión en $75 \%$, y después de incluir las nuevas técnicas de intervención estas variables volvieron a disminuir 21 y $25 \%$, respectivamente, al contrastar las sesiones siete y nueve.

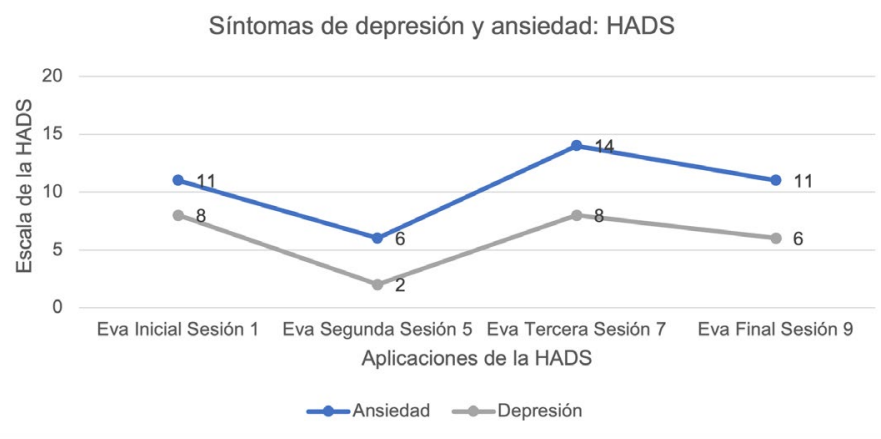

Figura 3.

Resultados en ansiedad y depresión: HADS.

\section{Relevancia social del cambio}

Se considera que la solución de problemas y la planeación de actividades puede utilizarse como técnica de intervención para modificar variables cognitivas atribuibles a un problema en particular; en este caso, una percepción negativa y poco funcional de la enfermedad, la cual limita el proceso de adaptación a la misma y tiene repercusiones emocionales. Si bien la solución de problemas se usó para tener una apreciación más amplia de las dificultades de la paciente y que la planeación de actividades fungiera como el proceso de solución, ambas estrategias se ajustaron de manera adecuada.

Se sabe que la planeación de actividades pretende cambios a nivel conductual (Kanter et al., 2010; Lejuez, Hopko y Hopko, 2001) y no a nivel cognitivo (Barraca, 2016); sin embargo, en este caso es evidente que el proceso de intervención fue modificando la percepción de Sandra acerca de su enfermedad, así como la función de dicha percepción para impactar de modo negativo en las repercusiones emocionales y de funcionalidad.

Así, es evidente que la percepción negativa de la enfermedad se asociaba de modo funcional con diversos sucesos presentes e históricos relacionados con un poco efectivo control del asma; evidencia mostrada por Moreno-San-Pedro y Roales-Nieto, (2003). En este contexto, Sandra estaba expuesta a diversas situaciones adversas respecto de su enfermedad (limitaciones y consecuencias negativas de la enfermedad y experiencia de síntomas), mismas que favorecían repercusiones en su estado emocional al estar relacionadas de manera con- 
tingente (Lewinsohn y Graf, 1973; Lewinsohn, Gotlib y Hautzinger, 2007; Nezu, Nezu y Lombardo, 2006).

Con el presente caso se evidencia que las consecuencias emocionales de la enfermedad pueden ser reguladas por la manera en que se percibe ésta. Dicha percepción se deriva de experiencias negativas previas (Diefenbach y Leventhal, 1996; Lugo-González, Pérez-Bautista, Robles-Montijo y Vega-Valero, 2019); por tanto, la evaluación de la percepción de enfermedad es una opción viable para proponer formas de abordaje dirigidas a su modificación e impactar en el estado emocional.

Se sabe que una percepción negativa de la enfermedad se va a relacionar con la ejecución de conductas desadaptativas para el automanejo de la enfermedad y estados emocionales negativos (Bahçecioğlu y Çevikakyl, 2014; Halm, Mora y Leventhal, 2006; Jessop et al., 2004; Kaptein, Klok, Moss-Morris y Brand, 2010; Lugo-González et al., 2014), de aquí la importancia de su intervención con técnicas cognitivas y conductuales.

Se comenta acerca del proceso de ajuste a la enfermedad, mismo que se ve acompañado de diversas barreras. En este caso los resultados durante la intervención sugerían avances en términos conductuales porque Sandra retomó diversas áreas de su vida que había dejado de lado. A nivel cognitivo, también se observaban mejorías con una modificación de la percepción de enfermedad y en el contexto emocional con una disminución en la sintomatología de ansiedad y depresión. Sin embargo, es evidente que alteraciones importantes como un cambio en el pronóstico de la enfermedad y una modificación radical en el abordaje médico, son capaces de revertir los logros a nivel psicológico.

También es evidente que la continuidad con la intervención favoreció de nuevo un proceso de cambio en las variables de desenlace, lo que comprueba que el afrontamiento de las problemáticas asociadas a la enfermedad es una ruta necesaria para su solución y mejoría de los pacientes con asma (Barton, Clarke, Sulaiman y Abramson, 2003; Ritz et al., 2013; Smith y Jones, 2015; Nezu et al., 2006; Sansom-Daly, Peate, Wakefield, Bryant y Cohn, 2012).

Dadas las relaciones identificadas en la evaluación conductual y los resultados de la intervención, este tipo de abordaje sería recomendable en el contexto clínico con pacientes con asma, considerándolos siempre como intervenciones complementarias en un contexto multidisciplinario, que si bien las evidencias de investigación no son fuertes, las instituciones encargadas a nivel internacional del manejo del asma las recomiendan cada vez más (GINA; 2019; Kew et al., 2016; Pateraki et al., 2018; Ritz et al., 2013; Smith y Jones, 2015; Yorke et al., 2015). Aunque sería necesario, para estudios futuros, obtener más evidencia si los cambios en la percepción de la enfermedad y variables psicológicas generan mejores resultados en el control del asma, como se ha mostrado en otras intervenciones de tipo cognitivo conductual (Guillen y Lugo-González, 2014; Kew et al., 2016; Lugo-González, Reynoso-Erazo y Fernández-Vega, 2016; Ritz et al., 2013).

\section{Pronóstico}

\section{Descripción de la fase de seguimiento}

Como se describió en el proceso de intervención, en la fase de seguimiento se continuó evaluando la percepción de enfermedad y el estado de ánimo. Sin embargo, dadas las circunstancias en que se encontraba Sandra, la psicoeducación, solución de problemas, la planeación de actividades y la revaloración cognitiva se enfocaron hacia el afrontamiento del nuevo diagnóstico y tratamiento.

\section{Resultados en la fase de seguimiento}

Si bien fue posible minimizar el impacto negativo en la percepción de enfermedad y en el estado emocional, no se logró regresar al nivel de la evaluación en la sesión cinco. Después de estas sesiones ya no se pudo dar seguimiento a la paciente debido al proceso de evaluación y tratamientos médicos complementarios que iniciaría; sin embargo, se auguró un resultado positivo por la manera en que consiguió los primeros resultados y al continuar efectuando las estrategias llevadas a cabo en la intervención, se esperaría que de modo progresivo mejorara a nivel emocional.

\section{Referencias}

Achstetter, L. I., Schultz, K., Faller, H., \& Schuler, M. (2016). Leventhal's common-sense model and asthma control: Do illness representations predict success of an asthma rehabilitation? Journal of Health Psychology, 1, 1-10. https://doi.org/10.1177/1359105316651332

Bahçecioğlu, G., \& Çevikakyl, R. (2014). Determination of effect on asthma control of illness perception of asthma patients. Acta Medica Mediterranea, 30, 591-600.

Barley, E., \& Lawson, V. (2016). Health psychology: Supporting the self-management of long-term conditions. British Journal of Nursing, 25(20), 1102-1107. https://doi. org/10.12968/bjon.2016.25.20.1102

Barraca, M. J. (2016). La activación conductual en la práctica: Técnicas, organización de la intervención, dificultades y variantes. Análisis y Modificación de Conducta, 42(165-6). http://dx.doi.org/10.33776/amc.v42i165-66.2792

Barragán, B., Parra, C., Contreras, M., \& Pulido, P. (2003). Manejo de la ansiedad mediante la combinación de desensibilización sistemática, con relajación muscular, relajación autógena y biorretroalimentación. Suma Psicológica, 10(2), http://dx.doi.org/10.14349/ sumapsi2003.97 
Estudio de caso | Intervención Cognitivo-Conductual para disminuir depresión...| Lugo-González \&Z Pérez-Bautista

Barton, C., Clarke, D., Sulaiman, N., \& Abramson, M. (2003). Coping as a mediator of psychosocial impediments to optimal management and control of asthma. Respiratory Medicine, 97(7), 747-761. https://doi.org/10.1016/ S0954-6111(03)00029-5

Beck, A. T., Rush, A. J., Shaw, B. F., \& Emery, G. (2010). Terapia cognitiva de la depresión. 19a. España: Descleé de Brouwer.

Bravo-González, M. C. (2014). Generalidades psicológicas de los padecimientos crónico-degenerativos. En L. Reynoso y A. L. Becerra (eds.). Teoría y práctica de la medicina conductual (pp. 43-64). México: Qartuppi.

Broadbent, E., Petrie, K., Main, J., \& Weinman, J. (2006). The brief illness perception questionnaire. Journal of Psychosomatic Research. 60, 631-637. https://doi. org/10.1016/j.jpsychores.2005.10.020

Cardiel, R. (1994). La medición de la calidad de vida. En L. Moreno, F. Cano-Valle y H. García-Romero (eds.). Epidemiología clínica (pp. 189-199). México: Interamericana-McGrawHill.

Ciprandi, G., Schiavetti, I., Rindone, E., \& Ricciardolo, F. L. (2015). The impact of anxiety and depression on outpatients with asthma. Annals of Allergy, Asthma \& Immunology, 115(5), 408-414. http://dx.doi.org/10.1016/j.anai.2015.08.007

Coban, H., \& Aydemir, Y. (2014). The relationship between allergy and asthma control, quality of life, and emotional status in patients with asthma: a cross-sectional study. Allergy, Asthma \& Clinical Immunology, 10(1), 67. https:// doi.org/10.1186/s13223-014-0067-4

Colom, F. (2011). Psicoeducación, el litio de las psicoterapias. Algunas consideraciones sobre su eficacia y su implementación en la práctica diaria. Revista Colombiana de Psiquiatría, 40. https://doi.org/10.1016/S00347450(14)60200-6

DeGood, D., Crawford, A., \& Jongsma, A. (1999). Asthma. En D. DeGood, A. Crawford, A. Jongsma (aut.). The behavioral medicine planner (pp.29-36). EUA: John Wiley and Sons.

Diefenbach, M. A., \& Leventhal, H. (1996). The commonsense model of illness representation: Theoretical and practical considerations. Journal of Social Distress and the Homeless, 5(1), 11-38. https://doi.org/10.1007/ BF02090456

Di Marco, F., Verga, M., Santus, P., Giovannelli, F., Busatto, P., Neri, M., Girbino, G., Bonini, S., \& Centanni, S. (2010). Close correlation between anxiety, depression, and asthma control. Respiratory Medicine, 104, 22-28. https://doi. org/10.1016/j.rmed.2009.08.005

Espinosa, F., Parra, M., Segura, N., Toledo, D., Menez, D., Sosa E., \& Torres, A. (2006). Ansiedad y depresión en asmáticos adultos en comparación con sujetos sanos. Revista Alergia México, 53(6), 201-206.

Foster, J. M., Lavoide, K. L., \& Boulet, L. P. (2011). Treatment adherence and psychosocial factors in severe asthma. En K. F. Chung, E. H. Bel y S. E. Wenzel (eds.). European Respiratory Monograph: Dificult to treat severe asthma. European Respiratory Society 51 (pp. 28-49). https://doi. org/10.1183/1025448x.erm5110

Freitas, P. D., Xavier, R. F., Passos, N. F., Carvalho-Pinto, R. M., Cukier, A., Martins, M. A., \& Carvalho, C. R. (2019). Effects of a behaviour change intervention aimed at increasing physical activity on clinical control of adults with asthma: Study protocol for a randomised controlled trial. BMC Sports Science, Medicine and Rehabilitation, 11(1), https://doi.org/10.1186/s13102-019-0128-6

Galindo, V. O., Benjet, C., Juárez-García, F., Rojas-Castillo, E., Riveros-Rosas, A., Aguilar-Ponce, J. L., Álvarez-Avitia, M. A., \& Alvarado-Aguilar, S. (2015). Propiedades psicométricas de la Escala Hospitalaria de Ansiedad y Depresión (HADS) en una población de pacientes oncológicos mexicanos. Salud Mental, 38(4), 253-258. https://doi.org/10.17711/ SM.0185-3325.2015.035

García-Flores, R., \& Sánchez-Sosa, J. J. (2013). Efectos de la entrevista motivacional en el tratamiento de la diabetes mellitus tipo 2. Psicología y Salud, 23(2), 183-193. https:// doi.org/10.25009/pys.v23i2.500

Gerontoukou, E. I., Michaelidoy, S., Rekleiti, M., Saridi, M., \& Souliotis, K. (2015). Investigation of anxiety and depression in patients with chronic diseases. Health Psychology Research, 3(2). https://doi.org/10.4081/hpr.2015.2123

Global Initiative for Asthma (GINA) (2019). Global strategy for asthma management and prevention. Recuperado de www.ginasthma.org

Grau-Abalo, J. A. (2016). Factores psicosociales y enfermedades crónicas: El gran desafío. Revista Latinoamericana de Medicina Conductual, 6(2), 103-112.

Guillen, D. B. C., González, C. R. A. L. M., Sánchez, C, C. T., \& Fernández-Vega, M. (2018). Escala de Ansiedady Depresión Hospitalaria (hads) en mexicanos con asma: Confiabilidad y validez. Psicología Iberoamericana, 26(2), 75-87. http:// www.redalyc.org/articulo.oa?id=133959841009

Guillen, D. B. C., \& González, C. R. A. L. M. (2019). Propiedades psicométricas del Inventario de Ansiedad de Beck en adultos asmáticos mexicanos. Psicología y Salud, 29(1), 5-16. https://doi.org/10.25009/pys.v29i1.2563

Guillen, D. B. C., \& Lugo-González, I. V. (2014). Tratamiento psicológico del asma en el campo de la medicina conductual. En L. Reynoso y A. L. Becerra (eds.). Teoría y Práctica de la Medicina Conductual (pp. 237-262). México: Qartuppi.

Halm, E., Mora, P., \& Leventhal, H. (2006). No Symptoms, No Asthma. The acute episodic disease beliefs is associated with poor self-management among inner-city adults with persistent asthma. Chest, 129, 573-580. https://doi. org/10.1378/chest.129.3.573

Iwakabe, S., \& Gazzola, N. (2009). From single-case studies to practice-based knowledge: Aggregating and synthesizing case studies. Psychotherapy Research, 19(4-5), 601-611. https://doi.org/10.1080/10503300802688494

Jessop, D. C., Rutter, D. R., Sharma, D., \& Albery, I. P. (2004). Emotion and adherence to treatment in people with asthma: An application of the emotional Stroop paradigm. British Journal of Psychology, 95(2), 127-147. https://doi. org/10.1348/000712604773952386

Kanfer, F, H., \& Saslow, G. (1965). Behavioral analysis: An alternative to diagnostic classification. Archives of General Psychiatry, 12, 529-538. PMID: 14286879. https://doi. org/10.1001/archpsyc.1965.01720360001001

Kanter, J., Manos, R., Bowe, W., Baruch, D., Baruch, A., \& Rusch, 
L. (2010). What is behavioral activation? A review of the empirical literature. Clinical Psychology Review, 30, 608620. https://doi.org/10.1016/j.cpr.2010.04.001

Kaptein, A., Klok, T., Moss-Morris, R., \& Brand, P. (2010). Illness perceptions: Impact on self-management and control in asthma. Current Opinion in Allergy and Clinical Immunology, 10, 194-199. https://doi.org/10.1097/ ACl.0b013e32833950c1

Karekla, M., Karademas, E. C., \& Gloster, A. T. (2019). The Common Sense Model of Self-Regulation and Acceptance and Commitment Therapy: Integrating strategies to guide interventions for chronic illness. Health Psychology Review, 13(4). https://doi.org/10.1080/17437199.201 $\underline{8.1437550}$

Katon, W. J. (2011). Epidemiology and treatment of depression in patients with chronic medical illness. Dialogues in Clinical Neuroscience, 13(1), 7. https://www.ncbi.nlm.nih. gov/pubmed/21485743

Kew, K. M., Nashed, M., Dulay, V., \& Yorke, J. (2016). Cognitive behavioural therapy (CBT) for adults and adolescents with asthma. Cochrane Database of Systematic Reviews, (9). https://doi.org/10.1002/14651858.CD011818.pub2

Larsen, D. P. (2009). Chronicity. En P. D. Larsen \& I. M. Lubkin (eds.). Chronic illness: Impact and intervention 7th edition (pp. 3-24). USA: Jones and Bartlett Publishers, LLC.

Lejuez, C., Hopko, D., \& Hopko, S. (2001). A brief behavioral activation treatment for depression: Treatment manual. Behavior Modification, 25(2), 255-286. https://doi. org/10.1177/0145445501252005

Leventhal, H., Leventhal, E. A., y Contrada, R. J. (1998). Selfregulation, health, and behavior: $A$ perceptual-cognitive approach. Psychology and Health, 13(4), 717-733. https:// doi.org/10.1080/08870449808407425

Lewinsohn, P. M., Gotlib, I. H., \& Hautzinger, M. (2007). Tratamiento conductual de la depresión unipolar. En V. Caballo (dir.). Manual para el tratamiento cognitivoconductual de los trastornos psicológicos (pp. 493-542). España: Siglo XXI.

Lewinsohn, P. M., \& Graf, M. (1973). Pleasant activities and depression. Journal of Consulting and Clinical Psychology, 41, 261-268. https://doi.org/10.1037/h0035142

Lugo-González, I. V., \& Pérez-Bautista, Y. Y. (2016). Intervención conductual para síntomas de depresión y ansiedad en un paciente con EPOC. Pensamiento Crítico: Revista de Investigación Multidisciplinaria, 3(5), 17-28. https:// udf.edu.mx/wp-content/uploads/2017/07/REVISTAAn\%CC\%830-3-No.5-2016-vol.-Il.pdf

Lugo-González, I. V., \& Pérez-Bautista, Y. Y. (2018). Planeación de actividades en pacientes con ERCT: Propuesta de intervención para depresión, ansiedad y calidad de vida. Revista Latinoamericana de Medicina Conductual. 8(2), 8-16. $\quad$ http://www.revistas.unam.mx/index.php/rlmc/ article/view/67376

Lugo-González, I. V., Pérez-Bautista, Y. Y., Robles-Montijo, S. S., \& Vega-Valero, C. Z. (2019). Representación de enfermedad y depresión en personas con ERCT y asma: Comparación por enfermedad. Psicología y Salud. 29(2), 237-247. http://psicologiaysalud.uv.mx/index.php/psicysalud/ article/view/2590
Lugo-González, I. V., Pérez-Bautista Y. Y., \& Sánchez-Cervantes, C. T. (2018). Validación de la escala de ansiedad y depresión hospitalaria en pacientes con ERC en hemodiálisis. En R. Díaz-Loving, I. Reyes-Lagunes y F. López-Rosales (eds.). Aportaciones actuales de la psicología social, volumen IV (pp. 1209-1225). México: AMEPSO.

Lugo-González, I. V., Reynoso-Erazo, L., \& Fernández-Vega, M. (2014). Percepción de enfermedad, depresión, ansiedad y control del asma: Una primera aproximación. Neumología y Cirugía de Tórax. 73(2), 114-121. https:// dx.doi.org/10.35366/51624

Lugo-González, I. V., Reynoso-Erazo, L., \& Fernández-Vega, M. (2016). Efecto de una intervención cognitivoconductual sobre ansiedad, depresión y severidad del asma en adultos. Revista Latinoamericana de Medicina Conductual. 6(1), 23-32. http://revistas.unam.mx/index. php/rlmc/article/view/58064

McAndrew, L. M., Musumeci-Szabó, T. J., Mora, P. A., Vileikyte, L., Burns, E., Halm, E. A., Leventhal, E., \& Leventhal, H. (2008). Using the common sense model to design interventions for the prevention and management of chronic illness threats: From description to process. British Journal of Health Psychology, 13(2), 195-204. https://doi. org/10.1348/135910708X295604

Morales, T., Alfaro, O., Sánchez, S., Guevara, U., \& Vázquez, F. (2007). Ansiedad y depresión por dolor crónico neuropático y nociceptivo. Revista Médica del Instituto Mexicano del Seguro Social, 46(5), 479-484. http://www. imbiomed.com.mx/1/1/

Moreno-San-Pedro, E. M., \& Roales-Nieto, J. G. (2003). El modelo de creencias de salud: Revisión teórica, consideración Moreno crítica y propuesta alternativa. I: Hacia un análisis funcional de las creencias en salud. International Journal of Psychology and Psychological Therapy, 3(1). https:// www.redalyc.org/pdf/560/56030105.pdf

Nezu, A. M., Nezu, C. M., \& D'Zurilla, T. J. (2007). Solving Life's Problems: A 5-Step Guide to Enhanced Well-Being. USA: Springer.

Nezu, A. M., Nezu, C. M., \& Lombardo, E. (2006). Formulación de casos y diseño de tratamientos cognitivo-conductuales: Un enfoque basado en problemas. México: Manual Moderno.

Nogueda, O, M, J., Pérez, T, B., Barrientos, C, V., Robles, G, R., \& Sierra, M, J. G. (2013). Escala de Ansiedad y Depresión Hospitalaria (HADS): Validación en pacientes mexicanos con infección por VIH. Psicología Iberoamericana, 21(2). http://www.redalyc.org/pdf/1339/133930525004.pdf

Pateraki, E., \& Morris, P. G. (2018). Effectiveness of cognitive behavioural therapy in reducing anxiety in adults and children with asthma: A systematic review. Journal of Asthma, 55(5), 532-554. https://doi.org/10.1080/02770 903.2017.1350967

Prapa, T., Porpodis, K., Fouka, E., Domvri, K., Zarogoulidis, P., Chasapidou, G., \& Papakosta, D. (2017). The role of psychological factors in the management of severe asthma in adult patients. Pneumon, 30(4). http://www. pneumon.org/assets/files/789/file697_528.pdf

Ritz, T., Meuret, A. E., Trueba, A. F., Fritzsche, A., \& Von Leupoldt, A. (2013). Psychosocial factors and behavioral medicine 
Estudio de caso | Intervención Cognitivo-Conductual para disminuir depresión... | Lugo-González \& Q Pérez-Bautista

interventions in asthma. Journal of Consulting and Clinical Psychology, 81(2), 231. https://doi.org/10.1037/ a0030187

Ryu, Y., Chun, E., Lee, J., \& Chang, J. (2010). Prevalence of depression and anxiety in outpatients with chronic airway lung disease. The Korean Journal of Internal Medicine, 25(1), 51-57. https://doi.org/10.3904/kjim.2010.25.1.51 Sansom-Daly, U. M., Peate, M., Wakefield, C. E., Bryant, R. A., \& Cohn, R. J. (2012). A systematic review of psychological interventions for adolescents and young adults living with chronic illness. Health Psychology: Official Journal of the Division of Health Psychology, American Psychological Association 31(3), 380. https://doi.org/10.1037/ a0025977

Smith, H. E., \& Jones, C. J. (2015). Psychological interventions in asthma. Current Treatment Options in Allergy, 2(2), 155168. https://doi.org/10.1007/s40521-015-0051-3
Stanescu, S., Kirby, S. E., Thomas, M., Yardley, L., \& Ainsworth, B. (2019). A systematic review of psychological, physical health factors, and quality of life in adult asthma. NPJ Primary Care Respiratory Medicine, 29(1), 1-11. https:// doi.org/10.1038/s41533-019-0149-3

Vázquez, F. (2002). La técnica de solución de problemas aplicada a la depresión mayor. Psicothema, 14(1), 516522. Recuperado de http://www.redalyc.org/

Yorke, J., Fleming, S., Shuldham, C., Rao, H., \& Smith, H. E. (2015). Nonpharmacological interventions aimed at modifying health and behavioural outcomes for adults with asthma: A critical review. Clinical \& Experimental Allergy, 45(12), 1750-1764. https://doi.org/10.1111/cea.12511

Zigmond, A., \& Snaith, R. (1983). The Hospital Anxiety and Depression Scale. Acta Psiquiatrica Scandinavica, 67, 361-370. PMID: $6880820 . \quad$ https://doi. org/10.1111/j.1600-0447.1983.tb09716.x 


\section{Meta-Análisis del Artículo}


Estudio de caso | Intervención Cognitivo-Conductual para disminuir depresión...| Lugo-González \&Z Pérez-Bautista

\section{Dimensión Cuantitativa}

\section{Perfil de Evaluación entre pares}
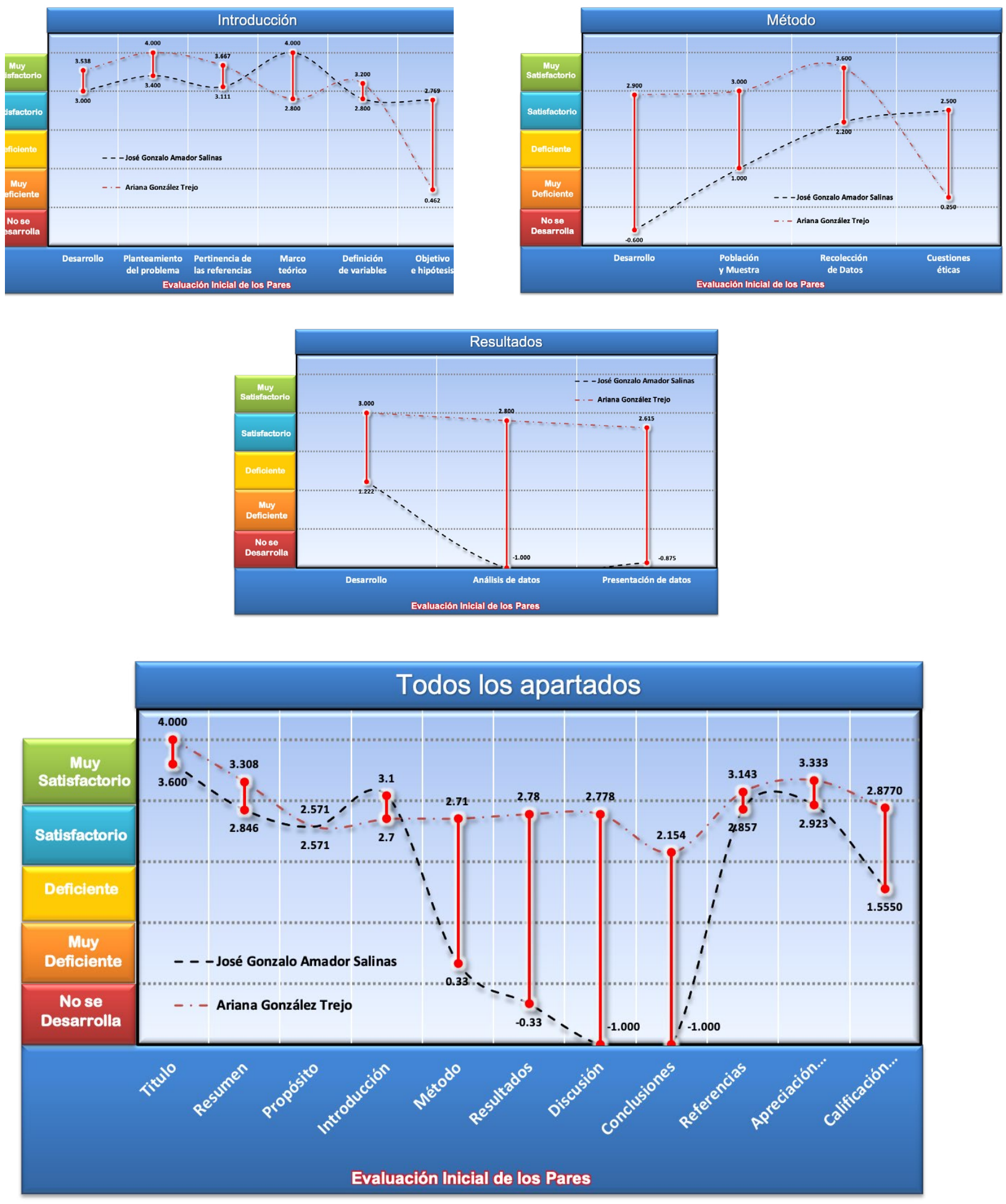


\section{Índice de Concordancia}

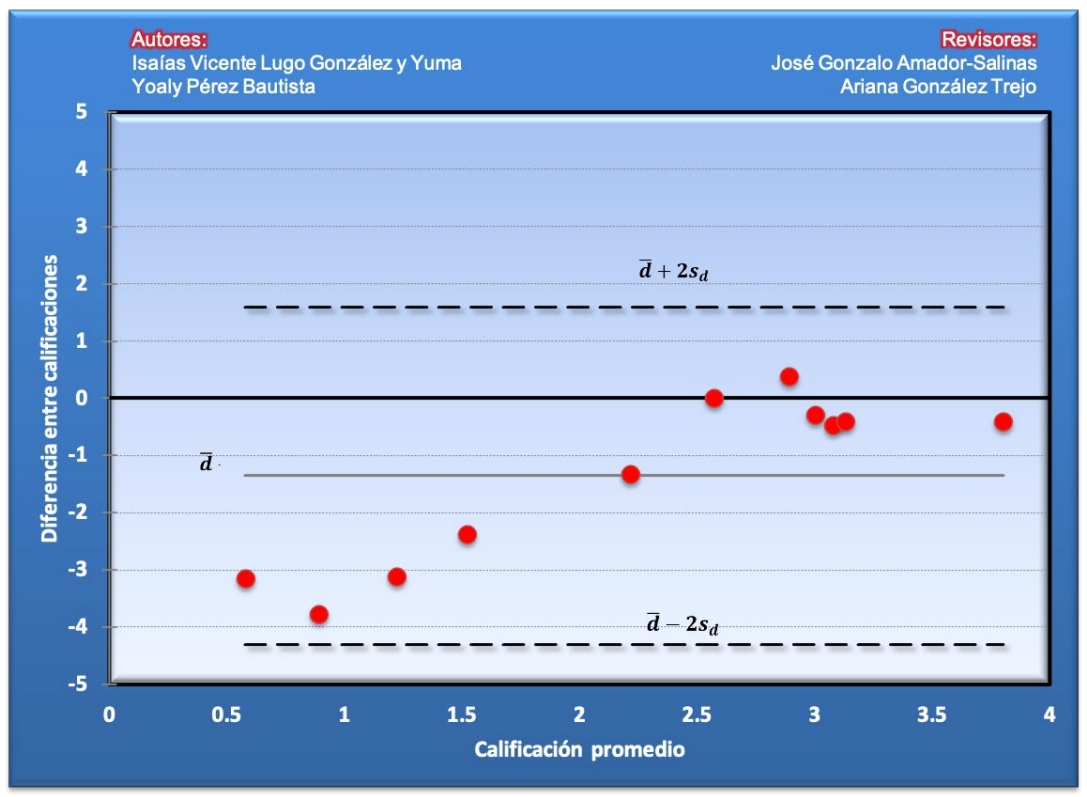

\section{Índice de Acuerdo}
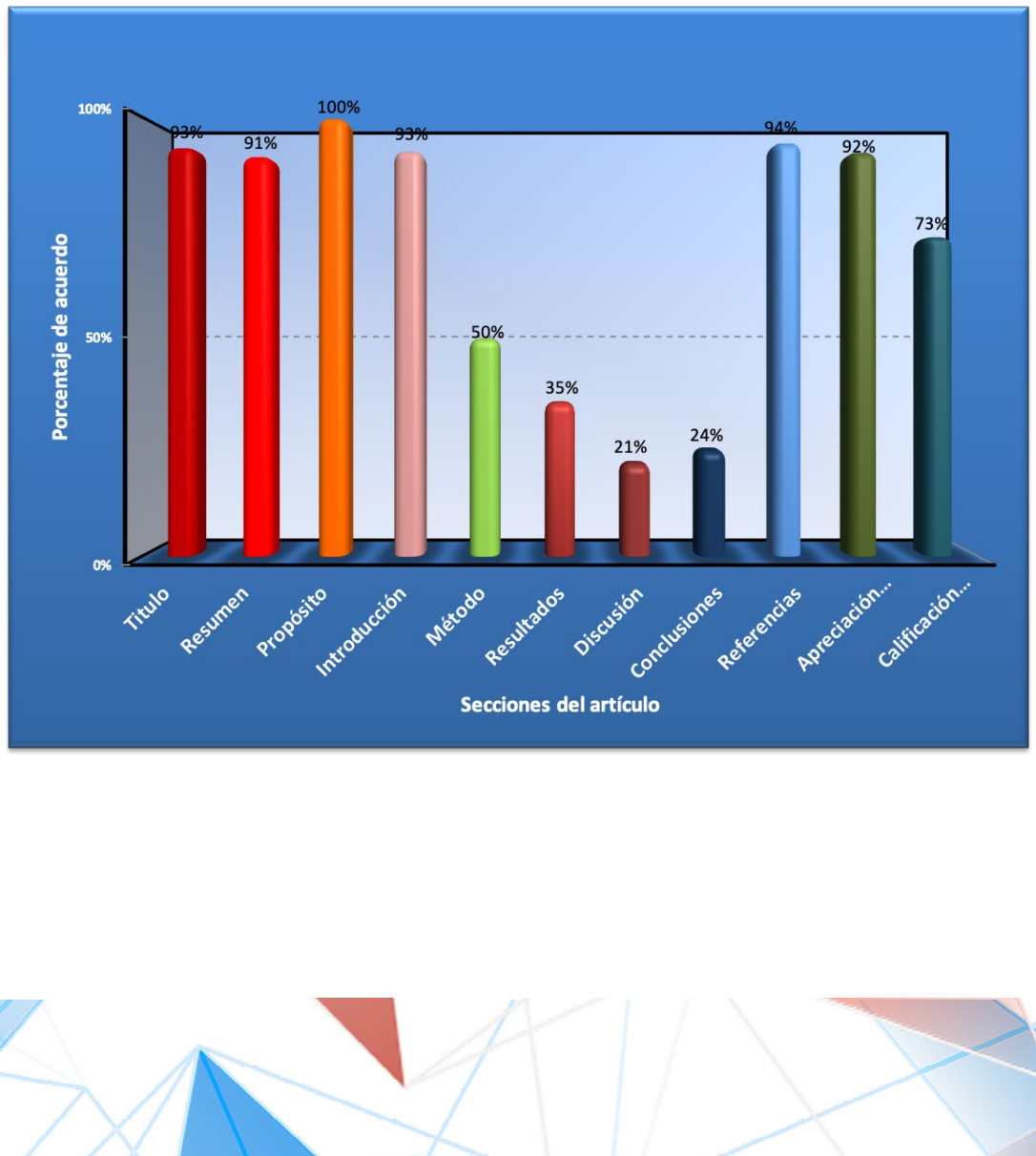


\begin{tabular}{|c|c|}
\hline Revisor 1 & Revisor 2 \\
\hline José Gonzalo Amador Salinas & Ariana González Trejo \\
\hline \multicolumn{2}{|c|}{ Título/Autoría } \\
\hline $\begin{array}{l}\text { En el título se menciona un tratamiento cognitivo-con- } \\
\text { ductual, pero en el desarrollo del trabajo y en el aborda- } \\
\text { je del mismo, se destaca con más énfasis el tratamiento } \\
\text { cognitivo, por lo que parece que faltaría lo relacionado } \\
\text { al tratamiento conductual y su análisis (línea base, di- } \\
\text { seño y tipo, etc.) }\end{array}$ & $\begin{array}{l}\text { Me pareció muy claro y conciso el título. No tendría pro- } \\
\text { puestas de cambio. }\end{array}$ \\
\hline \multicolumn{2}{|c|}{ Resumen } \\
\hline $\begin{array}{l}\text { Hace falta que incluya información como el diseño de } \\
\text { la intervención (AB, ABA, etc.) y el proceso estadístico } \\
\text { con el que midió el impacto del tratamiento }\end{array}$ & $\begin{array}{l}\text { En relación a la cantidad de palabras, en el presente for- } \\
\text { mulario se indica un máximo de } 150 \text {; sin embargo en las } \\
\text { directrices indica un rango de } 150 \text { a 200, con lo cual el } \\
\text { presente resumen cumple con este criterio. Como punto a } \\
\text { mejorar sería mencionar como parte del método, las téc- } \\
\text { nicas utilizadas para la intervención, ya que sólo se men- } \\
\text { cionan los instrumentos de evaluación, pero no queda } \\
\text { claro qué técnicas se aplicaron para obtener los resultados } \\
\text { que mencionan. }\end{array}$ \\
\hline \multicolumn{2}{|c|}{ Próposito del Estudio } \\
\hline $\begin{array}{l}\text { Es importante que se aclare el diseño de la investigación } \\
\text { y la manera en que se verificará su impacto, con el uso } \\
\text { de la estadística para diseños } \mathrm{N}=1 \text {, o la comparación } \\
\text { de los resultados del pretest con el postest en el caso } \\
\text { de los cuestionarios aplicados. De esta manera habrá } \\
\text { relación entre el propósito con el método, resultados y } \\
\text { discusión. }\end{array}$ & $\begin{array}{l}\text { Fue complicado identificar el propósito del presente tra- } \\
\text { bajo. Sugiero en primer lugar una redacción más clara, } \\
\text { que facilite su identificación en el texto ya que se encuen- } \\
\text { tra después del plan de intervención. En segundo lugar, } \\
\text { sería agregar la hipótesis o pregunta de investigación, ya } \\
\text { que no se menciona de forma clara. }\end{array}$ \\
\hline \multicolumn{2}{|c|}{ Introducción } \\
\hline $\begin{array}{l}\text { El manuscrito es sin duda muy interesante, sería oportuno } \\
\text { que se incluyan datos que están señalados para que tuviera } \\
\text { mayor impacto }\end{array}$ & $\begin{array}{l}\text { Falta indicar la pregunta de investigación o hipótesis. } \\
\text { Respecto al marco teórico, se presenta de manera gene- } \\
\text { ral la eficacia de las intervenciones cognitivo-conductual. } \\
\text { Como sugerencia se pondría ampliar en la descripción } \\
\text { de algunas intervenciones que demuestren su eficacia y } \\
\text { vincularlas con la propuesta de intervención que se está } \\
\text { desarrollando. }\end{array}$ \\
\hline
\end{tabular}




\begin{tabular}{|l|}
\hline Revisor 1 \\
\hline \\
\hline \multicolumn{2}{|c|}{ Métod } \\
$\begin{array}{l}\text { Alguno de los puntos que se manejan como "no están } \\
\text { desarrollado" fortalecerán la argumentación científica }\end{array}$
\end{tabular}

De forma general cumple con los elementos necesarios del método; sin embargo se observa un tanto confusa la organización del método, este sería un punto a mejorar. También es importante aclarar, cómo se aseguró la confidencialidad de los datos del participante, es decir, si hubo un consentimiento informado o algún otro acuerdo que protegiera al participante.

\section{Resultados}

Fue complicado identificar en qué momento comienza la presentación de resultados. Sería importante una mejor organización que ayude al lector a identificar la parte de resultados. También respetar un orden en cuanto a la presentación de los mismos, iniciando con los datos positivos o aquellos que reflejan el objetivo planteado y posteriormente mostrar aquellas dificultades que se tuvieron.

\section{Discusión}

$\mathrm{Si}$ en el marco teórico se agregaran algunos estudios que

Desarrollar la sección muestren la eficacia de las técnicas empleadas, podría ayudar a tener más elementos para discutir los resultados obtenido en la propuesta de intervención que se desarrolló.

\section{Conclusiones}

Desarrollar la sección

Diferenciar las conclusiones de la discusión, ya que no está de manera clara y dificulta su identificación en el texto. Esto permitiría que de manera clara se observe la contribución del estudio.

\section{Referencias}

Sin comentarios

Diferenciar las conclusiones de la discusión, ya que no está de manera clara y dificulta su identificación en el texto. Esto permitiría que de manera clara se observe la contribución del estudio. 
